II. Die Bildung von Ozon, Wasserstoffsuperoxyd und Ueberschwefelsaure bei der Electrolyse verdünnter Schwefelsilure; von Franz Richarz.

(Hiersa Taf. III Pig. 8-6.)

Die erste auf die Bildung von Superoxyden ber der Electrolyse verdünnter Schwefelsäure zwischen Platinelectroden bezügliche Beobachtung datirt von Faraday ${ }^{1}$ ), welcher fand, dass unter Umständen der entwickelte Sauerstoff weniger als die Hälfte des Volumens des Wasserstoffs einnahm. Er schrieb diesen Verlust auf Bildung von Wasserstoffsuperoxyd. Demnächst entdeckte Schönbein ${ }^{2}$ ) die Anwesenheit des von ihm Ozon genannten Körpers in dem entwickelten Sauerstoff und constatirte, dass grosse Stromdichtigkeit an der positiven Electrode, sowie Abkühlung des Electrolyten die Bildung desselben begünstigen. Seiner Ozontheorie gemäss nahm er an, dass eine dem Ozon gleiche Menge Antozon gebildet werde, welches sich mit dem Wasser zu Wasserstoffsuperoxyd vereinige. Meidinge ${ }^{3}$ ) hingegen wies nach, dass die Mengen des disponiblen Sauerstoffs in der Flüssigkeit viel grösser waren, als im Ozon. Nachdem Soret die Ozontheorie Schönbein's zu Fall gebracht, war auch die Annahme, dass äquivalente Quanta Ozon und Wasserstoffsuperoxyd gebildet würden, unnöthig. Weiterhin untersuchte C. Hoffman ${ }^{4}$ ), welche Mengen Ozon und disponiblen Sauerstoffs in dem mit Schwefelsäure versetzten Wasser bei rerschiedener Concentration gebildet werden. Die oxydirende Substanz in der Flüssigkeit hielt er noch für Wasserstoffsuperoxyd, obwohl er erkannte, dass sie in den meisten Fällen übermangansaures Kali erst nach längerem Stèhen entfärbt. Rundspaden $n^{5}$ ) gelangte zu dem Resultate, dass

1) Faraday, Exp. Res. 7. $\$ 728.1834$ und Phil. Trans. 124. p. 91. 1834.

2) Schönbein, Pogg. Ann. 50. p. 616. 1840.

3) Meidinger, Lieb. Ann. 88. p. 57. 1853.

4) C. Hoffmann;, Pogg. Ann. 139. p. 607. 1867.

5) Rundspaden, Lieb. Ann. 151. p. 306. 1869. 
ausser der Bildung von Ozon und Wasserstoffsuperoxyd noch eine andere Ursache des Sauerstoffverlustes existiren müsse. Berthelot endlich wies nach, dass diese in der Bildung der ron ihm entdeckten Ueberschwefelsäure zu suchen ist.

Der Anhydrid der Ueberschwefelsäure, dessen Zusammensetzung er analytisch und synthetisch zu $\mathrm{S}_{2} \mathrm{O}_{7}$ bestimmte ${ }^{2}$ ), erhielt er durch Einwirkung der electrischen Entladung in einer Ozonröhre auf Schwefligsäureanhydrid oder auf Schwefelsäureanhydrid in Sauerstoff; die Reaction wird ausgedruckt durch die Formeln:

$$
2 \mathrm{SO}_{2}+3 \mathrm{O}=\mathrm{S}_{2} \mathrm{O}_{7} \text { resp. } 2 \mathrm{SO}_{3}+\mathrm{O}=\mathrm{S}_{2} \mathrm{O}_{7} \text {. }
$$

Das so erhaltene Ueberschwefelsåureanhydrid löste Berthelot in verdunnter Schwefelsäure, wodurch zweifellos ein Hydrat gebildet wird, dessen Constitution nicht angegeben werden kann. Ganz dasselbe chemische Verhalten wie diese Lösung zeigt die unter geeigneten Umständen bei der Electrolyse von Schwefelsäure in ziemlicher Verdünnung an der positiven Electrode gebildete oxydirende Substanz ${ }^{2}$ ), welche demnach ein Hydrat der Ueberschwefelsäure ist. Berthel ot hat dann weiterhin ${ }^{3}$ ) eingehend die Eigenschaften der uberschwefelsäurehaltigen verdünnten Schwefelsäure untersucht, sowie Versuche gemacht uber die Bildung derselben und des Wasserstoffsuperoxyds bei der Electrolyse. In Bezug auf letztere Frage blieb noch zu beantworten, in welcher Weise eine Reihe ron physikalischen Umständen die Bildung der Superoxyde beeinflusst. Auf Veranlassung des Hrn. Geheimraths v. Helmholtz habe ich es unternommen, diese Untersuchung durchzuführen, und ich möchte es nicht unterlassen, meinem hochverehrten Lehrer an dieser Stelle für die Anregung zu den in vorliegender Arbeit beschriebenen Versuchen, sowie fur die freundliche Unterstützung bei Ausführung derselben meinen wärmsten und aufrichtigsten Dank zu sagen.

1) Berthelot, Ann. de chim. et de phys. (5) 14. p. 345.1878.

2) Berthelot, Ann. de chim. et de phys. (j) 14. p. 354.1878 und Compt. rend. 86. p. 71. 1878.

3) Berthelot, Compt. rend. 90. p. 269. 1880. 
I. Chemische Methoden.

Das Ozon wurde gemessen durch Abscheidung von Jod aus Jodkalium; das abgeschiedene Jod wurde nach Zusatz von Stärke mit einer Lösung von unterschwefligsaurem Natron titrirt. Die angewandten Reactionen sollen verlaufen nach den Formeln:

$$
\begin{gathered}
2 \mathrm{KJ}+\mathrm{O}_{3}=\mathrm{K}_{2} \mathrm{O}+\mathrm{O}_{2}+2 \mathrm{~J} \\
2 \mathrm{~S}_{2} \mathrm{O}_{3} \mathrm{Na}_{2}+2 \mathrm{~J}=\mathrm{S}_{4} \mathrm{O}_{6} \mathrm{Na}_{3}+2 \mathrm{NaJ} .
\end{gathered}
$$

Allein ohne Gegenwart einer Säuré bildet Ozon mit Jodkalium jodsaures Kalium. Es wurde daher vor jedem Versuch der Jodkaliumlösung ein wenig Salzsåure ${ }^{1}$ ) zugesetzt, und nach der Entfärbung durch Natriumhyposulfit durch weiteren Zusatz von Salzsäure controlirt, ob der ursprüngliche Zusatz genügt hatte, um die Bildung von jodsaurem Kalium zu verhindern.

Zur Bestimmung der Ueberschwefelsäure gibt Berthelot vier Reactionen an: die Oxydation vón Zinnchlorür, Eisenvitriol, schwefliger Säure und die Abscheidung von Jod aus Jodkalium. Nach mehrfachen Versuchen erwies sich Eisenvitriol als die sicherste und ohne umständliche Vorsichtsmaassregeln unwendbare Maasstlüssigkeit gegen Ueberschwefelsäure. Die Reaction ist folgende:

$$
2 \mathrm{SO}_{4} \mathrm{Fe}+\mathrm{SO}_{3}+\mathrm{O}=\left(\mathrm{SO}_{4}\right)_{3} \mathrm{Fe}_{2} \text {. }
$$

Es wird zu der Flüssigkeit, deren Gehalt an Ueberschwefelsäure bestimmt werden soll, ein bestimmtes Volumen (Stópselfläschchen) Eisenvitriollösung zugesetzt, dessen Titer durch normale Permangunatlösung vorher bestimmt ist. Der Verlust des Titer der Vitriollösung wird wieder durch dieselbe Permanganatlösung gemessen, deren Einwirkung nach der Formel geschieht:

$$
\begin{aligned}
2 \mathrm{KMnO}_{4}+10 \mathrm{FeSO}_{4} & +8 \mathrm{H}_{2} \mathrm{SO}_{4}=2 \mathrm{MnSO}_{4}+5 \mathrm{Fe}_{8}\left(\mathrm{SO}_{4}\right)_{3} \\
& +8 \mathrm{H}_{2} \mathrm{O}+\mathrm{K}_{2} \mathrm{SO}_{4} .
\end{aligned}
$$

In beiden Phasen der Bestimmung ist also die Anwesenheit von \$chwefelsăure nicht nur nicht störend, sondern sogar erforderlich. Die Oxydation des Eisenvitriols erfordert einige

1) Vgl. Mohr, Lehrbuch der Titrirmethode, 5. Aufl, p. 301. 
Zeit, weshalb man nicht sofort mit übermangansaurem Kali zurücktitriren darf, sondern etwa $3 / 4$ bis 1 Stunde warten muss. Bei Beobachtung dieser Vorschrift erhält man bei wiederholtem Titriren gleicher Volumina derselben überschwefelsäurehaltigen Lösung bis auf zehntel Procente gleichen Titer. Die Methode ist bei allen Concentrationen gleich zurerlässig.

In derselben Weise wie Ueberschwefelsäure wirkt nun aber auch Wasserstoffsuperoxyd auf Eisenvitriol. Eine käufliche wăsserige Lösung von Wasserstoffsuperoxyd ergab unmittelbar mit $\mathrm{KMnO}_{4}$ bis zur Entfärbung titrirt den Gehalt 31,5; wurde zu einem gleichen Volumen derselben Eisenvitriol zugesetzt und dann mit $\mathrm{KMnO}_{4}$ zurücktitrirt, so ergab sich der Gehalt zu 31,3. Die Bestimmung mit Eisenvitriol gibt mir also in den Fällen, wo die Flüssigkeit gleichzeitig Ueberschwefelsäure und Wasserstoffsuperoxyd enthält, die Gesammtmenge des disponiblen Sauerstoffs.

Das Wasserstoffsuperoxyd allein erhalte ich durch Titriren mit einer normalen Lösung von chemisch reinem übermangansauren Kalium:

$$
2 \mathrm{KMnO}_{4}+5 \mathrm{H}_{2} \mathrm{O}_{2}=2 \mathrm{MnO}+\mathrm{K}_{2} \mathrm{O}+5 \mathrm{H}_{2} \mathrm{O}+5 \mathrm{O}_{3} \text {. }
$$

Damit die Reaction vorschriftsmässig verlaufe, ist die Gegenwart von verdünnter Schwefelsäure erforderlich ${ }^{1}$ ), welche beim Titriren der der Electrolyse unterworfenen Flüssigkeit ja schon ron rornherein zugegen ist. Andererseits zersetzen concentrirte Sauren das Kaliumpermanganat ${ }^{2}$; es ist daher darauf zu achten, dass die Messung des Wasserstoffsuperoxyds stets in verdünnten Flüssigkeiten geschehe.

Je nach der Menge der electrolytisch gebildeten Superoxyde wurde entweder die gesammte Flussigkeit titrirt oder ein genau bestimmter Bruchtheil derselben. Eisenvitriollösungen von $1 / 2,1 / 20$ und $1 / 200$; Kaliumpermanganatlösungen von $1 / 20$ und $1 / 200$; Natriumhyposulfitlösung ron $1 / 80$ des Moleculargewichts in Grammen auf $1 \mathrm{l}$ genügten in allen Fällen, um die Analyse bequem und hinreichend genau zu machen.

1) Siehe Mohr, l. c. p. 175 .

2) Mohr, l. c. 
II. Physikalische Methoden.

Bereits Schönbein constatirte, dass zur Bildung erheblicher Mengen der Superoxyde bei der Electrolyse grosse Stromdichtigkeit an der positiven Electrode und niedrige Temperatur erforderlich seien. Um erstere zu erhalten, nahm ich zur positiven Electrode stets einen äusserst feinen Platindraht. Um constante niedrige Temperatur zu haben, wurde die Zersetzungszelle schon etwa eine Stunde vor jedem Versuch mit kleingestossenem Eis umgeben; damit die Flüssigkeit sich während der Zersetzung nicht erwärme, war es einestheils geboten, als Gefäss eine Röhre von kleinem Durchmesser zu nehmen, anderentheils während des Versuches das etwa abschmelzende Eis immer wieder an die Zersetzungszelle anzudrücken.

Um die Intensität constant erhalten zu können, wurde in denselben Stromkreis mit dem Zersetzungsgefäss ein Rheochord und eine Tangentenbussole eingeschaltet. Letztere wurde dann durch Verschiebung des Schlittens auf dem Rheochord während der Dauer eines Versuches stets auf der gewünschten Ablenkung erhalten.

Um mit Sicherheit bei allen Versuchen Schwefelsare von gleicher Concentration zu haben, wurde vorher ein grösseres Quantum - etwa $10 \mathrm{l}$ - Säure bereitet rom specifischen Gewicht 1,209, also ungefähr rom Maximum des Leitungsvermögens. Bei dieser Verdünnung wird unter keinen Umständen eine Spur $\mathrm{H}_{3} \mathrm{O}_{2}$ gebildet.

Von grossem Einfluss auf die Mengen der gebildeten Superoxyde ist die Vertheilung der Stromdichtigkeit auf der positiven Electrode. Damit dieselbe sich nicht von Versuch zu Versuch in uncontrolirbarer Weise andere, war Sorge zu tragen, dass die Electroden ihre Lage nicht änderu kunnten. $\mathrm{Zu}$ diesem $\mathrm{Z}$ wecke wurde der als positive Electrode dienende feine Draht mit dem einen Ende an eine Erhöhung der Glaswand angeschmolzen, das andere Ende um einen dickeren Platindraht, durch welchen der Strom zugeleitet wurde, gewickelt, mit Glas umsponnen und so in die Gefässwand eingesetzt, dass die Electrode möglichst straff gespannt war. 
Zur negativen Electrode wurde anfänglich, um den Widerstand der Zelle möglichst klein zu machen, ein Platinblech gewählt, welches durch zwei Drähte an den Enden festgehalten wurde. Es war nicht möglich; die beiden Electroden in einer Röhre von ca. $4 \mathrm{~cm}$ Durchmesser so unverruckbar einander gegenüber zu befestigen, dass nicht die Zahlen für die gebildete Ueberschwefelsäure bei mehreren mit gleichen Flüssigkeitsmengen, gleicher Dauer des Stromschlusses und gleicher Intensität angestellten Versuchen innerhalb weiter Grenzen schwankend geblieben wären.

Eine Veränderung in der Lage der Electroden wird auf die Vertheilung der Stromdichtigkeit auf denselben einen geringeren Einfluss haben, wenn sie in den Schenkeln einer U-förmigen Röhre angebracht sind. In der That wurden für eine solche die Mengen der bei ganz gleichen Versuchen gebildeten Ueberschwefelsäure etwas besser übereinstimmend. Die noch immer grossen Schwankungen waren wohl auf unregelmässige Veränderungen in der Zusammensetzung der Flussigkeit im positiven Schenkel zu schieben, hervorgebracht durch Diffusion der Ueberschwefelsäure in den anderen Schenkel, und durch die electrolytische Wanderung der Schwefelsäuremolecüle. Dass letztere in der That beträchtliche Störungen hervorruft, zeigt sich, wenn mit der U-Röhre Versuche von verschiedener Dauer des Stromschlusses bei gleicher Intensität gemacht werden:

\begin{tabular}{c|c|c}
\hline \hline $\begin{array}{c}\text { Dauer } \\
\text { des Strom- } \\
\text { schlusses }\end{array}$ & $\begin{array}{c}\text { Mengen der ge- } \\
\text { bildeten Ueber- } \\
\text { schwefelsare }\end{array}$ & Differenz \\
\hline $1 / 4$ St. & 2,13 & 2,13 \\
$1 / 2 "$ & 6,55 & 4,42 \\
$3 / 4 "$ & 10,56 & 4,01 \\
$1 / n$ & 14,94 & 4,38 \\
$8 / 4 "$ & 18,25 & 3,31
\end{tabular}

In der ersten Viertelstunde wird also weniger Ueberschwefelsăure gebildet, als in den năchstfolgenden; dies erklärt sich vollkommen aus der Wanderung der Schwefelsăure zum positiven Pol; denn bei 30 procentiger Säure wächst die Menge 
der ceteris paribus gebildeten Ueberschwefelsäure mit der Concentration, wie die späteren Versuche zeigen werden.

Aus diesen Gründen musste eine U-Röhre verworfen und zur einfachen Röhre zurückgekehrt werden. Um den Einfluss von Lagenveränderungen der Electroden auf die Vertheilung der Stromdichtigkeit auf denselben zu vermindern, wurden dieselben ubereinander angebracht. Die negative Electrode, als welche noch immer ein Platinblech diente, war die untere, damit durch die grössere von unten her aufsteigende Gasmenge die ganze Flüssigkeit möglichst lebhaft umgerührt werde. Ein solches Gefäss gab bei aufeinanderfolgenden Versuchen von gleicher Dauer des Stromschlusses, gleicher Intensitat, mit gleichen Volumen Saure zwar nicht mehr in weitem Intervall regellos schwankende, aber regelmăssig abnehmende Zahlen für die gebildete Ueberschwefelsäure. Dieselben waren für eine Viertelstunde Stromschluss in der Reihenfolge, wie die Versuche angestellt wurden:

$$
16,40 ; 16,24 ; 15,70 \text {, }
$$

und nachdem inzwischen Versuche bei längerer Dauer des Stromschlusses gemacht worden waren, war die Zahl für eine Viertelstunde nur mehr:

\section{4,16 .}

Es musste also eine fortschreitende Veränderung an den Electroden durch die Electrolyse selbst hervorgebracht werden; denn die benutzte Säure war immer unverăndert von derselben Beschaffenheit. Meine Aufmerksamkeit richtete sich zuerst auf die negative Electrode, welche sich bekanntlich mit Wasserstoff beladet. Es wäre ja denkbar gewesen, dass durch die zunehmende Wasserstoffbeladung des Platinbleches dasselbe eine zunehmende reducirende Kraft auf die Ueberschwefelsäure erlangt bätte. Um eine solche zu verringern, wurde zunächst als negative Electrode ebenfalls ein feiner Draht genommen. Dies hatte keine Wirkung; auch bei diesem Zersetzungsgefass nahmen die Zahlen für die gebildete Ueberschwefelsăure bei ganz gleichen aufeinanderfolgenden Versuchen fortschreitend ab. Sauerstoffentwickelung an der negativen Electrode und lebhaftes galranisches Ausgluhen derselben hatte ebenfalls keinen Erfolg. Wurde 
nun aber die positive Electrode ausgeglüht, so ergab ein folgender in gleicher Weise wie die früheren angestellter Versuch wieder den Anfangswerth für die Menge der gebildeten Ueberschwefelsăure. Die störende Veränderung ging also an der positiven Electrode vor sich und war durch Ausglühen zu beseitigen.

Um das letztere bequem auf galvanischem Wege bewerkstelligen zu können, wurden dem feinen Drahte ron beiden Seiten Zuleitungen von aussen gegeben, indem seine Enden auf dickere Platindrähte aufgewickelt, diese mit leichtflussigem Glas umsponnen und in die Wand der Glasröhre eingesetzt wurden. So erhielt das Zersetzungsgefäss die zu den vergleichenden Versuchen benutzte Gestalt wie sie in Fig. 3 gezeichnet ist. Wie schon bemerkt, ist die positive Electrode die obere. Um vor Nebenschliessung durch das Schmelzwasser des umgebenden Eises sicher zu sein, ist die Zuleitung zur negativen Electrode in eine dünne Glasröhre $r$ eingeschmolzen. Ein- und Ausfüllung der Flüssigkeit, deren Volumen bei allen Versuchen das gleiche war, geschah durch das Rohr $e$ vermittelst Saugen, resp. Blasen von $k$ aus. Nachdem die Flüssigkeit und die darüber befindliche Luft im Gefässe sich bis auf $0^{\circ}$ abgekühlt, wurde die Einfüllungsröhre $e$ zugestőpselt, dann die Röhre $k$ durch die eine Bohrung eines doppelt durchbohrten Kautschukpfropfens gesteckt, welch letzterer eine mit Jodkaliumlösung gefüllte weitere Röhre verschliesst, und durch dessen andere Bohrung das electrolytische Gas, nachdem es durch die Jodkaljumlösung gestrichen ist, zu einer Gasmessröhre weiter geleitet wird. Die mit Glaswolle gefüllte Kugel $g$ hat den Zweck, mitgerissene Säure zurückzuhalten.

Um ein Springen des Glases zu verhüten, geschah das galvanische Ausglühen der positiven Electrode in der Weise, dass ihre Temperatur durch Ausschalten ron Widerstand im Stromkreise allmählich gesteigert wurde. Durch wenige Versuche ergab sich, dass einige Minuten lebhaften Rothgluhens genugen, um die fragliche Veränderung zu beseitigen. Bei 3 Versuchen von viertelstündiger Dauer des Stromschlusses 
bei ganz gleichen Verhältnissen waren, wenn nach jedem Versuche die positive Electrode ausgeglitht wurde:

$$
148,9 ; 151,0 ; 150,0 \mathrm{ccm}
$$

der $1 / 200$ Permanganatlösung ăquivalent der Menge der gebildeten Ueberschwefelsäure. Bei denselben Versuchen war das entwickelte Ozon äquiralent:

$$
0,90 ; 1,18 ; 0,92 \mathrm{ccm}
$$

der $1 / 60$ Natriumhyposulfitlösung. Es ist ersichtlich, dass die Zahlen viel unsicherer sind, als diejenigen fur die Ueberschwefelsäure. Die obigen Zahlen für das entwickelte Ozon sind mit $0,8 \mathrm{zu}$ multipliciren, um mit den angegebenen für die Ueberschwefelsäure direct rergleichbar zu sein; erstere sind gegen letztere sehr klein. Die Unsicherheit in der Ozonbestimmung ist daher für die Beurtheilung des ganzen electrolytischen Vorganges ohne Bedeutung.

\section{Vrsache der Veränderung der positiven Electrode.}

Es wurde zunächst eine Anzahl von Versuchen angestellt, um die Ursache der räthselhaften Verănderung der positiven Electrode zu ermitteln. Vielleicht bewirkte dieselbe der an ihr vorbeistreichende Wasserstoff. Um diesen fernzuhalten, wurde deshalb als negative Electrode einmal ein in das Rohr e gesteckter Platindraht verwandt, und nach vorherigem Ausglühen bei Benutzung derselben Electrode als positiver 15 Stunden lang bei Zimmertemperatur zersetzt; dann ohne vorheriges Glühen mit frischer Săure wieder ein den auf dieser Seite oben angeführten Versuchen gleicher Versuch gemacht. Derselbe ergab für die Ueberschwefelsäure nur: 129,5 .

Der vorbeistreichende Wasserstoff bringt die Veränderung also nicht hervor.

Flüchtige Verunreinigungen, z. B. niedere Oxydationsstufen des Stickstoffes, welche rom Bleikammerprocess her häufig in geringer Menge in der Schwefelsäure vorhanden zu sein plegen, würden keine $\nabla$ erănderung der Electrode hervorbringen können; sie üben aber - wenn sie vorhanden waren - uberhaupt keine merkbare Einwirkung auf die Ueberschwefelsăure ans; denn ein Versuch mit vorher längere 
Zeit zum Sieden erhitzter, dann wieder aufs ursprüngliche specifische Gewicht gebrachter Säure ergab wie bei den anderen Versuchen: $\quad 149,6$.

Etwaige Verunreinigungen der Säure durch Salze von Mangan oder Blei würden an der positiven Electrode einen störenden Ueberzug ihrer Superoxyde geben. Ich würde jene Salze entfernen, wenn ich die zu benutzende Säure schon vorher längere Zeit der Electrolyse unterwerfe. Dies geschah 2 Tage hindurch zwischen Platinplatten, wobei keine Ueberschwefelsäure gebildet wurde. Dann wurde nach vorherigem Ausglühen der positiven Electrode 4 Stunden lang bei Zimmertemperatur diese vorbehandelte Säure zersetzt. Auch dann war die fragliche Veränderung eingetreten; denn ein folgender Normalversuch mit der gewöhnlichen Säure ergab ohne vorheriges Ausglühen nur:

130,4

für die gebildete Ueberschwefelsäure.

Ferner wurde nun untersucht, ob bei der Electrolyse anderer Säuren ebenfalls die Veränderung der positiven Electrode eintrat. Nach vorherigem Ausglühen wurde an derselben 17 Stunden lang aus verdünnter Essigsäure bei Zimmertemperatur Sauerstoff entwickelt. Ein Normalversuch mit der zu allen anderen Versuchen benutzten verdünnten Schwefelsäure gab dann ohne vorheriges Ausglühen:

139,6 .

Wurde ganz analog verdünnte Salpetersäure zersetzt, so war die Menge der gebildeten Ueberschwefelsäure bei einem folgenden Normalversuch auch nur:

$$
136,7 \text {. }
$$

Jedesmal, wenn die Kleinheit des gebildeten Quantums der Ueberschwefelsăure bewies, dass die Veränderung an der positiven Electrode eingetreten war, wurde durch Ausglühen mit folgendem Normalversuch controlirt, ob die Veränderung wieder beseitigt war; die Menge der gebildeten Ueberschwefelsäure lag dann immer zwischen 149,0 und 151,0 .

Aus diesen Versuchen glaube ich den Schluss ziehen zu dürfen, dass die Veränderung der positiven Electrode, welche bei unter ganz gleichen Umständen angestellten Versuchen 
die Menge der gebildeten Ueberschwefelsäure kleiner werden lässt, nur dadurch hervorgebracht wird, dass die positive Electrode überhaupt als solche dient, dass uberbaupt Sauerstoff an ihr entwickelt wird.

Im Folgenden wurde stets zwischen zwei Versuchen die positive Electrode ausgeglüht, was von nun an nicht mehr weiter angeführt werden soll.

IV. Reduction von Ueberschwefeläure an der negativen Electrode.

C. Hoffmann und Berthelot machen ohne weiteres die allerdings sehr wahrscheinliche Annahme, dass Ueberschwefelsäure und Wasserstoffsuperoxyd, wenn sie zur negativen Electrode gelangen, dort theilweise reducirt werden, und umgeben deshalb die positive Electrode mit einem porösen Thongefäss. Die electroly tische $W$ anderung der Schwefelsüure im Verein mit der electrischen Endosmose bringen dann fortschreitende Veränderung der Concentration und der Menge der im Thoncylinder vorhandenen Flüssigkeit hervor, weshalb eine ähnliche Trennung der Electroden für meine Versuche unzulässig war.

Reduction von Ueberschwefelsäure an der negativen Electrode macht auch noch ein ihrem Sauerstoff äquivalentes Quantum Wasserstoffgas verschwinden, wird also ein erhebliches Dificit an nachweisbaren electrolytischen Producten hervorbringen. Um ein solches constatiren zu können, musste der Reductionsfactor der Tangentenbussole, deren Ablenkung während der Dauer eines Versuches constant erhalten wurde, mit möglichster Genauigkeit bestimmt werden. Zuverlässig ist nur die experimentelle Bestimmung; dieselbe geschah für verschiedene Ablenkungen der Bussole, wobei noch die Correction wegen der Nadellänge zu berucksichtigen war, mit dem sqnst zur Entwickelung der Superoxyde benutzten Zersetzungsgefäss, indem dasselbe in ein Wasserbad von ca. $90^{\circ}$ gesetzt wurde. Es bildeten sich dann nur verschwindende Spuren von Ozon und Ueberschwefelsäure. Zugleich war Absorption von Knallgas in der verdunnten Schwefelszure Ann. d. Phys. n. Chem. N. E. XXIV. 
ausgeschlossen. Die sich ergebenden $W$ erthe des Reductionsfactors waren bis auf $1 / \% \%$ einander gleich.

Dass thatsächlich Ueberschwefelsäure durch nascirenden Wasserstoff reducirt wird, zeigen folgende Versuche. Säure ron bekanntem Gehalt an Ueberschwefelsäure wurde eine gemessene Zeit hindurch mit constanter Intensität in einem Voltameter mit Platinplatten der Electrolyse unterworfen. Das Volumen der Flüssigkeit in diesem Gefässe war klein (etwa $20 \mathrm{ccm}$ ), sodass etwaige Absorption ron Knallgas nur sehr unbedeutend sein konnte. Nach Beendigung der Zersetzung hatte dann der Titer der Flüssigkeit erheblich abgenommen. Aus dieser Abnahme war leicht zu berechnen, wie viel Cubikcentimeter $W$ asserstoffgas zur Reduction verbraucht worden waren.

Aus der Ablenkung der Tangentenbussole berechnete sich das entwickelte Knallgasvolumen $\mathrm{zu}$. . . . . . . . . . . . . . .

Wirklich entwickelt wurden - reducirt auf $0^{\circ}, 760 \mathrm{~mm}$ und Trockenheit . . . . . . . 88,63"

$Z_{\mathbf{a}}$ der gemessenen Reduction von Ueberschwefelsäure waren an Wasserstoffgas erfor-

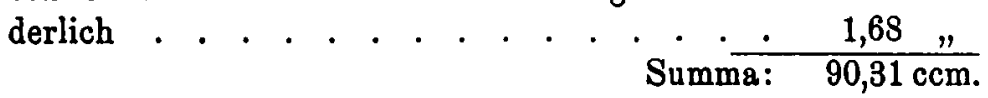

Ein zweiter Versuch wurde in derselben Weise mit Flüssigkeit von bedeutend höherem Gehalte an Ueberschrefelsäure angestellt.

Es wurde gemessen:

Entwickeltes Gas . . . . . . . . $162,27 \mathrm{ccm}$.

Verschwundenes Wasserstoffgas aus der Reduction von Ueberschwefelsäure berechnet . . 18,52 , Summa: $180,79 \mathrm{ccm}$.

Die Ablenkung der Tangentenbussole ergab 178,63 "

Werden nun während der Dauer eines Versuches Superoxyde gebildet, so ist zu dem Volumen des wirklich entwickelten Gases das auf $0^{\circ}$ und $760 \mathrm{~mm}$ reducirte Volumen zu addiren, welches der in den nachweisbaren Superoxyden enthaltene disponible Sauerstoff in gasförmigen Zustande 
einnehmen würde. Gegenüber dem aus der Tangentenbussole berechneten Knallgasvolumen bleibt dann immer noch ein Deficit, welches um so kleiner ist, je höher die Temperatur, bei welcher zersetzt wurde. Dies zeigen folgende bei nahezu gleicher Intensität und einviertelstündigem Stromschluss angestellten Versuche, von denen die drei ersten zugleich einen Maassstab bieten, inwieweit die in Betracht kommenden Grössen constant erbalten werden konnten.

\begin{tabular}{|c|c|c|c|c|c|c|}
\hline $\begin{array}{c}\text { Temperatur } \\
\text { des Zersetzungsgefuisses }\end{array}$ & $\begin{array}{c}\text { I. } \\
\text { Versuch }\end{array}$ & $\begin{array}{c}0^{0} \\
\text { II. } \\
\text { Versuch }\end{array}$ & versuch & $\begin{array}{l}\text { ca. } 20^{\circ} \\
\text { Iv. } \\
\text { Versuch }\end{array}$ & $\begin{array}{l}\text { ca. } 50^{\circ} \\
v . \\
\text { versuch }\end{array}$ & $\begin{array}{l}\text { ca. } 75^{\circ} \\
\text { VI. } \\
\text { Versuch }\end{array}$ \\
\hline \multirow{3}{*}{ 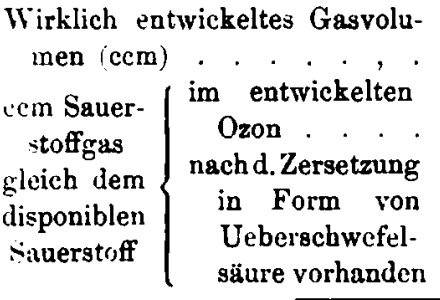 } & 71,60 & 71,15 & 71,46 & 77,47 & $צ 9,22$ & 89,89 \\
\hline & 0,10 & 0,11 & 0,11 & 0,10 & 0,08 & 0,04 \\
\hline & 16,11 & 16,38 & 16,21 & 11,93 & 0,29 & 0,07 \\
\hline Summa: & 87,36 & 57,64 & 87,78 & $\$ 9,50$ & 89,59 & 90,00 \\
\hline \multirow{3}{*}{$\begin{array}{l}\text { Aus der Ablenkung der Tangen- } \\
\text { tenbussole berechnetes Knall- } \\
\text { gasvolumen. . . . . . . }\end{array}$} & & & & & & \\
\hline & 89,97 & 90,07 & 90,07 & 90,93 & 90,36 & 90,21 \\
\hline & 2,11 & 2,43 & 2,29 & 1,43 & 0,77 & 0,21 \\
\hline
\end{tabular}

Das Deficit kann sowohl von theilweiser Reduction der Weberschwefelsäure an der negativen Electrode als auch von Absorption des Knallgases herrühren. Beide werden mit steigender Temperatur vermindert; erstere deshalb, weil weniger Ueberschwefelsäure gebildet wird. Es sei bemerkt, dass ein dem Volumen der verdünnten Säure $(91,4 \mathrm{ccm})$ gleiches Volumen destillirtes Wasser bei derselben Temperatur $\left(0^{9}\right)$ und $760 \mathrm{~mm}$ Barometerstand $1,8 \mathrm{ccm}$ Wasserstoff und $3,8 \mathrm{ccm}$ Sauerstoff absorbiren könnte. Im Folgenden wird in jedem einzelnen Falle zu untersuchen sein, welcher Vorgang überwiegend das Deficit hervorruft. Rührt dasselbe überwiegend ron Reduction der Ueberschwefelsäure her, so ist ein Drittel desselben zu der nach dem Versuch in der 
Flüssigkeit vorhandenen Menge Ueberschwefelsäure hinzuzuaddiren, um die an der positiven Electrode gebildete Menge Ueberschwefelsäure zu erhalten.

Die obigen Versuche ergeben zugleich, wie die Bildung der Superoxyde bei sonst gleichen Umständen von der Temperatur abhängig ist. Bemerkenswerth ist, dass die Mengen der gebildeten Ueberschwefelsäure ausserordentlich viel schneller mit wachsender Temperatur abnehmen, als die des Ozons.

V. Abhängigkeit der Bildung der Superoxyde von der Dauer des Stromschlusses.

Welche Function der Dauer des Stromschlusses unter sonst constant bleibenden Verhältnissen der Gehalt der Flüssigkeit an Ueberschwefelsäure ist, lässt sich angeben, wenn ich die denkbar einfachsten Annahmen über die Bildung der Ueberschwefelsäure an der positiven und ihreReduction an der negativen Electrode mache. In Bezug auf erstere nehme ich an, dass die im Zeitelement $d t$ gebildete Menge proportional sei der Menge der zur Zeit $t$ vorhandenen, noch nicht in Ueberschwefelsäure verwandelten Schwefelsäure. Ferner nehme ich an, dass die am negativen $P_{0}$ reducirte Menge proportional sei dem augenblicklichen Gehalt der Flüssigkeit an Ueberschwefelsäure. Der Zuwachs des Gehaltes der Flüssigkeit in derselben Zeit ist die Differenz der an der positiven Electrode gebildeten, und an der negativen zerstörten Ueberschwefelsäure. Nenne ich $S$ die Menge der ursprünglich vorhandenen Schwefelsäure, $M_{1}$, resp. $M_{2}$ die bis zur Zeit $t$ an der Sauerstoffelectrode gebildete, resp. an der Wasserstoffelectrode reducirte, $\boldsymbol{M}$ die zur Zeit $t$ in der Flüssigkeit vorhandene Menge Ueberschwefelsäure, gemessen durch die in ihr enthaltene Schwefelsäure, $p$ und $q$ zwei positive Constante, so soll also sein:

$$
\begin{gathered}
\frac{d M_{1}}{d t}=p(S-M), \\
\frac{d M_{9}}{d t}=q M \\
\frac{d M}{d t}=\frac{d M_{1}}{d t}-\frac{d M_{9}}{d t}=p S-(p+q) M
\end{gathered}
$$


Ich führe zwei neue positive Constante $A$ und $n$ ein, indem ich setze:

$$
p+q=n ; \quad p S=A n .
$$

Wenn ich die Zeit von dem Augenblicke des Stromschlusses an rechne, in welchem die Flüssigkeit noch keine Ueberschwefelsäure enthält, so ergibt die Integration der Differentialgleichung (3):

$$
M=A\left(1-e^{-n t}\right) .
$$

Die Form des Ausdrucks für $M$ bleibt dieselbe, wenn ich $M$ in anderen Einheiten ausdrücke, z. B. durch das Volumen, welches der in der Ueberschwefelsäure enthaltene disponible Sauerstoff als Gas bei $0^{\circ}$ und $760 \mathrm{~mm}$ einnehmen würde.

Für kleine Zeiten ergibt die Entwickelung der Exponentialfunction, wenn ich nur das lineare Glied berücksichtige: $\boldsymbol{M}=A n t$,

also Proportionalität mit der Dauer des Stromschlusses; für grosse Zeiten nähert sich $M$ asymptotisch dem Werthe $A$.

Sehen wir zu, inwieweit die Formel mit der Wirklichkeit im Einklang ist. Die folgenden Versuche von verschiedener Dauer des Stromschlusses sind angestellt bei nahezu derselben Intensität, wie jene auf p. 195. Die fünfte Reihe "Deficit" hat dieselbe Bedeutung wie dort. Die angegebenen

\begin{tabular}{|c|c|c|c|c|c|c|c|}
\hline \multirow[b]{2}{*}{ 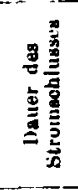 } & \multirow[b]{2}{*}{ 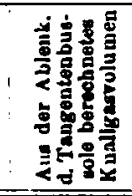 } & \multirow{2}{*}{\multicolumn{2}{|c|}{ 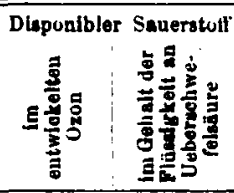 }} & \multirow[b]{2}{*}{ 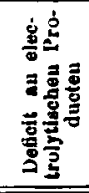 } & \multicolumn{3}{|c|}{ Zunabme auf jo "t St. berechnet } \\
\hline & & & & & 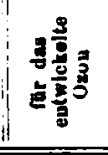 & 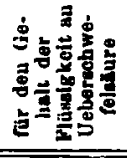 & 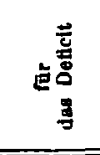 \\
\hline $1 . \mathrm{St}$. & 90,02 & 0,11 & 16,25 & 2,28 & 0,11 & 16,25 & 2,28 \\
\hline $1_{1 / 2}$, & 180,96 & 0,33 & $32, \overline{3} \mathrm{i}$ & 4,33 & 0,22 & 16,32 & 2,05 \\
\hline 1 & 361,9 & 0,9 & 60,2 & 11,8 & 0,28 & 13,8 & 3,74 \\
\hline 2 & 722.2 & 1,9 & 109,6 & 31,5 & 0,25 & 12,3 & 4,93 \\
\hline
\end{tabular}
Zahlen sind Cubikcentimeter.

In der Annahme, dass bei einer grősseren Intensităt das Maximum des Gehaltes an Ueberschwefelsăure schneller zu erreichen sei, wurde diese Versuchsreihe abgebrochen und die folgende angestellt. 


\begin{tabular}{|c|c|c|c|c|c|c|c|}
\hline \multirow[b]{2}{*}{ 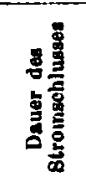 } & \multirow[b]{2}{*}{ 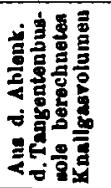 } & \multicolumn{2}{|c|}{ Disponibler Ssuerstoff! } & \multirow[b]{2}{*}{ 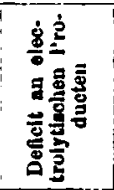 } & \multicolumn{3}{|c|}{ I Zunabme auf jo $1 /$ st berechnet } \\
\hline & & 玉竎苞 & 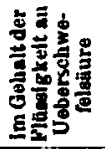 & & 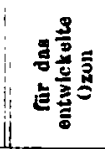 & 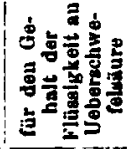 & 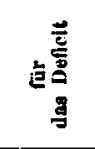 \\
\hline $1 / \mathrm{St}$. & 197,11 & 0,61 & 33,96 & 4,52 & 0,61 & 33,96 & 4,52 \\
\hline & 1375 & 6,00 & 199,0 & 91,0 & 0,7 & 29,6 & 12,4 \\
\hline 4 & 3150 & 11,3 & 308,4 & 879 & 0,66 & 13,7 & 23,5 \\
\hline 7 & 5524 & 23,0 & 393,9 & 742 & 0.97 & 7,1 & 38,6 \\
\hline 8 & 6313 & 26,2 & 406,9 & 905 & 0.3 & $+3,2$ & 40,8 \\
\hline 9 & $\tau 103$ & 30,4 & 402,0 & 1073 & 1,0 & $-1,2$ & 42,0 \\
\hline
\end{tabular}

In beiden Reihen war die Intensität, wie die aus der Ablenkung der Tangentenbussole berechneten Knallgasvolumina zeigen, bei den einzelnen Versuchen nicht immer genau dieselbe. Die Schwankungen, die dadurch in den übrigen Zahlen hervorgerufen sein können, fallen in den Bereich der Beobachtungsfehler.

Die erste Reihe zeigt in den beiden ersten Versuchen wie verlangt für kleine Zeiten Proportionalität des Gehaltes der Flüssigkeit mit der Dauer des Stromschlusses. Die zweite Versuchsreihe zeigt die wesentliche Abweichung von der theoretischen Formel, dass der Gehalt schliesslich wieder abnimmt. Es ist dies offenbar die Folge jener Veränderung der positiven Electrode, welche bei aufeinander folgenden gleichen Versuchen die Menge der gebildeten Ueberschwefelsäure immer kleiner werden lässt, und welche hei langerem Stromschlusse während desselben schon sehr beträchtlich werden muss. Infolge dieser Veränderung wird der End. zustand nicht der sein, dass in gleicher Zeit gleichviel Ueberschwefelsäure am positiven Pol gebildet und am negativen reducirt wird; sondern es wird schliesslich weniger an jenem entwickelt, als an diesem zerstört werden und daher der Gehalt der Flüssigkeit wieder abnehmen.

Die Constante $n$ der theoretischen Formel ist aus allen Combinationen je zweier Versuche zu berechnen; als Gewicht einer solchen Einzelbestimmung kann die Zeitdifferenz der beiden Beobachtungen gelten. Nit dem gefundenen $n$ gibt jeder Versuch einen Werth für $A$; von diesen wird das 
arithmetische Mittel genommen. Für die erste Versuchsreihe ergibt sich in dieser Weise, eine Viertelstunde als Zeiteinheit genommen:

$$
M=261,0\left(1-e^{-0,08597 t}\right),
$$

woraus sich für die vier beobachteten Zeiten die Werthe ergeben würden:

$$
\text { Beobachtet ist: } \begin{aligned}
16,65-32,26-60,37-107,04 . \\
16,25-32,57-60,2-109,6 .
\end{aligned}
$$

Für die $z$ weite Reihe wird, wenn ich die Constanten nur aus den ersten 5 Versuchen berechne:

$$
M=443,3\left(1-e^{-0,0764 t}\right) \text {; }
$$

welche Formel für die 6 Beobachtungen ergeben vürde:

$$
32,63-202,6-312,7-391,1-404,8-415 .
$$

Beobachtet wurde: $33,96-199-308,4-393,9-406,9-40$.

In beiden Fällen ist die Uebereinstimmung eine ziemliche, bis auf die letzte Zahl der zweiten Versuchsreihe.

Setze ich den gefundenen Ausdruck für $M$ als Function von $t$, welcher jedenfalls den wirklichen Gehalt der Flüssigkeit ziemlich richtig angibt, in die Differentialgleichung (2) auf p. 196, so erhalte ich durch Integration bei der getroffenen Wahl des Anfangspunktes der Zeit:

$$
M_{2}=\eta\left(A t-\frac{M}{n}\right)
$$

Würde das Deficit an nachweisbaren electrolytischen Producten nur durch Reduction von Ueberschwefelsäure erzeugt sein. so wäre ein Drittel desselben gleich $\boldsymbol{M}_{2}$. Mit rorstehender Formel könnte ich dann $q$ berechnen. Es würde sich bei der ersten Versuchsreihe ergeben:

$$
\begin{aligned}
& \text { für } t=\begin{array}{lll}
1 & 2 & 4
\end{array} \quad 8 \text { Viertelstunden, } \\
& q=0,087 \quad 0,0 \pm 3 \quad 0,030 \quad 0,023
\end{aligned}
$$

Der Umstand, dass die so gewonnenen Werthe für $q$ im Anfange viel grösser sind, lässt sich vollkommen durch die Annahme erklären, dass das Deficit zum Theil und anfänglich zum grössten Theil durch Absorption von Knallgas verursacht ist. Der letzte Werth ron $q$ kommt dem richtigen jedenfalls am năchsten. 
Bei der zweiten Versuchsreihe wird:

$$
\text { fiur } \begin{array}{rlcccc}
t & =1 & 8 & 16 & 28 & 32 \text { Viertelstunden, } \\
q & =0,093 & 0,0339 & 0,0310 & 0,0338 & 0,03+0 .
\end{array}
$$

Bei den letzten vier Beobachtungen war offenbar das Deficit schon weit überwiegend auf Reduction zurückzuführen, daher die Werthe für $q$ ziemlich gleich sind; das Mittel derselben ist:

$$
q=0,0332 \text {. }
$$

Die Constante $p$ lässt sich berechnen aus der Definitionsgleichung:

$$
p S=A n \text {. }
$$

Hierin will ich als Werth von $A n$ denjenigen nehmen, der aus der Anwendung der für kleine Zeiten geltenden Gleichung:

$$
M=A n t
$$

folgt. Aus den beiden ersten Beobachtungen der Reihe 1 ergibt sich: $A n=16,27$

aus der ersten der Reihe 2 :

$$
A n=33,96 \text {. }
$$

Ferner ist $S$, wenn ich für $A$ die bisherige Einheit beibehalte, die Anzahl Cubikcentimeter Sauerstoffgas bei $0^{0}$ und $760 \mathrm{~mm}$ Barometerstand, welche erforderlich sind, um die ganze vorhandene Schwefelsäure zu Ueberschwefelsäure zu oxydiren. Es berechnet sich aus der Anzahl Gramm $\mathrm{SO}_{3}$ in der Säure, und der Molecularformel $\mathrm{S}_{2} \mathrm{O}_{7}$ der Ueberschwefelsäure jener Werth:

Die Gleichung:

$$
S=1783 \mathrm{ccm} \text {. }
$$

$$
p S=A n
$$

ergibt dann:

für die 1. Versuchsreihe $p=0,00912$,

fur die 2. Versuchsreihe $p=0,0190$.

Nun soll nach seiner Definition sein $n=p+q ; n$ ist aber auch schon direct bestimmt aus den Werthen von $\boldsymbol{M}$. Es ist:

\begin{tabular}{c|c|c}
\hline \hline & für die 1. Reihe für die 2. Reihe \\
\hline \hline direct bestimmt: & 0,066 & 0,0764 \\
$p+q$ & 0,032 & 0,0526
\end{tabular}

$p+q$ ist also beidemal sehr viel kleiner als $n$. Diese Abweichung erklärt sich durch jene fatale Veränderung an der positiven Electrode, deren Eintritt während der Dauer eines 
Versuches nicht verhindert werden kann; infolge derselben wird $M$, der Gehalt der Flüssigkeit an Leberschwefelsäure, sich schneller einem niedrigeren Maximum nähern, $d$. h. es wird sich die Constante $n$ aus den Werthen von $M$ grösser, $A$ kleiner ergeben, als wenn die Electrode unverändert bliebe. In die directe Bestimmung von $p$ gehen dagegen nur die Beobachtungen bei kurzer Dauer des Stromschlusses ein; bei denselben ist die fragliche Veränderung der positiven Electrode noch unbedeutend.

Wenn es möglich wäre, die Veränderung der positiven Electrode zu verhindern, also die unseren Voraussetzungen entsprechenden Werthe für $n$ und $A$ zu erhalten, ferner die an der negativen Electrode reducirten Ueberschwefelsäurequanta und damit die Constante $q$ hinreichend sicher zu bestimmen, so würde ich aus den beiden Definitionsgleichungen :

$$
n=p+q, \quad p S=A n,
$$

die Constante $S$ erhalten als das Volumen Sauerstoffigas, welches die ganze vorhandene Schwefelsäure zu Ueberschwefelsäure oxydirt. Andererseits kenne ich den Werth von $S$ direct ausgedrückt in Grammen Schwefelsäureanhydrid. Aus diesen beiden Werthen für $S$ liesse sich die Molecularformel der Ueberschwefelsäure berechnen. Meine Versuche lassen dies infolge der angegebenen Mängel nicht zu.

In Bezug auf den Verlauf der Ozonentwickelung zeigt sich, dass die Menge des in der ersten Viertelstunde entwickelten Ozons scheinbar in beiden Versuchsreihen kleiner ist, als die in jeder folgenden Viertelstunde entwickelte Menge. Es ist dies aber eine Folge davon, dass nach jedem Versuch ein Theil des entwickelten Ozons, ohne durch das Jodkalium gestrichen zu sein, in dem Raume über die Flüssigkeit im Zersetzungsgefässe zurlickbleibt. Der dadurch herrorgerufene Verlust fällt bei den Differenzen verschiedener Versuche aus. Die in allen folgenden Viertelstunden gebildeten Ozon. mengen schwanken sehr, können aber wohl als gleich betrachtet werden.

Für verschiedene Zersetzungsgefasse von gleicher Gestalt, wie das zu meinen Versuchen benutzte, ist, wie durch Versuche bestatigt wurde, bei sonst gleichen Umständen der 
Maximalgehalt der Flüssigkeit an Ueberschwefelsäure um so kleiner und muss um so schneller erreicht werden, je geringer die Stromdichtigkeit an der negativen Electrode ist, da dann die Ueberschwefelsäure an einer grösseren Fläche mit nascirendem Wasserstoff in Berührung kommt und bei gleichem Gehalt der Flüssigkeit in gleicher Zeit mehr reducirt wird.

\section{Einfluss der Stromdichtigkeit.}

Verhalten sich bei meiner Zersetzungszelle, bei welcher die Lage der Electroden vollkommen fixirt ist, bei zwei Versuchen von kurzer Dauer die Intensitäten wie $1: n$, die Zeiten des Stromschlusses wie $n: 1$, sodass bei beiden Versuchen gleiche Quanta Wasser zersetzt werden, so werden sich in Bezug auf die entwickelten Superoxyde die beiden Versuche so rerhalten, als ob ich beidemal dieselbe Intensität und die gleiche Dauer des Stromschlusses habe, aber die Strom. dichtigkeit an allen Stellen der Electroden - es kommt hier nur auf die positive an - im Verhältnisse von 1:n vergrössert hätte. Für Versuche ron gleicher und kleiner Dauer des Stromschlusses bei einer Reihe ron verschiedenen Intensitäten werden sich daher die Quotienten der entwickelten Superoxyde durch die Intensitäten verhalten wie die Mengen der Superoxyde, welche gebildet sein würden bei verschiedenen je der betreffenden Intensität proportionalen Werthen der mittleren Stromdichtigkeit auf der positiven Electrode, wenn aber Intensität und Dauer des Stromschlusses bei allen Versuchen die gleichen wären.

Die Resultate einer derartigen Versuchsreihe gibt die Tabelle (p. 203) wieder. Die Dauer des Stromschlusses war jedesmal 1/, Stunde.

Von der Intensität an, welche $22.43 \mathrm{~cm}$ in der Viertelstunde lieferte, abwärts wurde eine empfindlichere Tangentenbussole ron grosser Windungszabl benutzt, deren Reductionsfactor, wie auf p. 193, experimentell bestimmt wurde. Bei den drei kleinsten Intensitäten konnte die gebildete Ueberschwefelsăure nicht mehr aus Versuchen ron nur einer Viertelstunde Stromschluss bestimmt werden; die obigen Zahlen sind daher aus Versuchen ron resp. $1 / 2$ Stunde, $1 \frac{1}{2}$ Stunde, 
3 Stunden Dauer durch Division durch resp. 2, 6. 12 erhalten. Auch so kommt beim letzten Versuch die Menge der zu bestimmenden Ueberschwefelsäure schon sehr nahe an die Grösse des Versuchsfehlers.

\begin{tabular}{|c|c|c|c|c|c|}
\hline $\begin{array}{l}\text { I. } \\
\text { Aus d. Ablenkung } \\
\text { d. Tangentenbues. } \\
\text { berechnotes } \\
\text { Knallgesrolumen } \\
\text { cem } \\
\end{array}$ & $\begin{array}{c}\text { II. } \\
\text { Disponibler } \\
\text { Sanerstoff im } \\
\text { ontwickelten } \\
\text { Ozon }\end{array}$ & 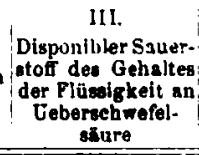 & $\begin{array}{c}\text { IV. } \\
\text { Deficit an } \\
\text { electrolytinch. } \\
\text { Producten }\end{array}$ & $\begin{array}{l}\text { r. } \\
\text { Quotient: } \\
\text { III. I. }\end{array}$ & $\begin{array}{c}\text { VI. } \\
\text { Quotient: } \\
\text { III+'t, IV. }\end{array}$ \\
\hline 285 & 1,1 & $45,1^{\circ}$ & 5.84 & 0,160 & 0,167 \\
\hline $19 \pi, 1$ & 0.61 & 34.00 & 4,52 & 0,173 & 0.180 \\
\hline 136,4 & 0,26 & 24.70 & 3.32 & 0.181 & 0,189 \\
\hline 90.02 & 0,11 & 16,25 & 2.28 & 0,181 & 0,130 \\
\hline 51,36 & 0,04 & $\$, \overline{0} s$ & $1,3 \dot{*}$ & $0,16:$ & 0,174 \\
\hline 20.43 & \multirow{5}{*}{ 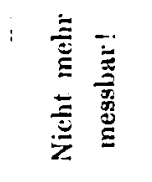 } & 2,32 & 0.36 & 0,104 & 0,109 \\
\hline 11.23 & & 0,40 & $0.3)$ & 0.035 & - \\
\hline $7, \mathbf{2 5}$ & & 0.03 & $0 . \overline{1} 5$ & $0.00 t$ & - \\
\hline 3,43 & & 0.0036 & 0.47 & 0.001 & - \\
\hline 1,80 & & 0.002 & 0.37 & 0,001 & - \\
\hline
\end{tabular}

Die Zahlen füs das entwickelte Ozon sind aus dem auf p. 201 angeführten Grunde alle zu klein. Erst die in den folgenden Viertelstiunden gemessenen Ozonmengen wären als richtig zu betrachten. Versuche für längere Dauer als eine Viertelstunde wurden bei folgenden Intensitäten gemacht.

\begin{tabular}{|c|c|c|c|}
\hline \multirow{2}{*}{$\begin{array}{l}\text { Aug der Ablenkung } \\
\text { d. Tangenteibubeole } \\
\text { berechnetes Knall- } \\
\text { gasrolumen fur } \\
\text { St. }\end{array}$} & \multicolumn{2}{|c|}{ Entwiokeltes Ozon } & \multirow{2}{*}{$\begin{array}{l}\text { Quotient der } \\
\text { dritten uod } \\
\text { ereten Ruhrik }\end{array}$} \\
\hline & $\begin{array}{l}\text { iu der ersten } \\
\text { Vierteletunde }\end{array}$ & $\begin{array}{l}\text { in jeder folxenden } \\
\vdots \quad \text { Viertoletunde }\end{array}$ & \\
\hline 90,02 & 0,11 & 0,25 & 0.0025 \\
\hline 197,3 & 0,61 & 0,85 & $0,00 \div 3$ \\
\hline $2 \$ 5$ & 1,1 & 1.5 & 0,0053 \\
\hline
\end{tabular}

Nach den gemachten Auseinandersetzungen sind die Quotienten in der 4. Rubrik proportional den Ozoumengen, welche bei sonst untereinander vollkommen gleichen Versuchen gebildet würden, wenn die mittlere Stromdichtigkeit auf der positiven Electrode den Zahlen der ersten Rubrik proportional wäre.

Die Rubrik IV der obigen Tabelle enthält das Deficit. welches die Summe: entwickeltes Gas + disponibler Sauerstoff in Ozon und Flussigkeit gegen die aus der Tangenten- 
bussole berèchnete Knallyasmenge bietet. Bei den kleineren Intensitäten ist dasselbe lediglich auf Absorption zurückzuführen, wenn man nicht die unnatürliche Annahme machen will, dass in einer Viertelstunde an der negativen Electrode mehr als $50 \mathrm{mal}$ soviel Ueberschwefelsäure reducirt worden sei, als der Gehalt der Flüssigkeit nach dem Versuch beträgt. Dass das Deficit bei kleinen Intensitäten weit überwiegend auf Rechnung der Absorption zu setzen ist, beweisen auch folgende Versuche von verschiedener Dauer des Stromschlusses.

\begin{tabular}{|c|c|c|c|c|}
\hline $\begin{array}{c}\text { Daner des } \\
\text { Stromseh' nases }\end{array}$ & $\begin{array}{c}\text { Ans der Ablen- } \\
\text { knng der Tan- } \\
\text { gentenbuseolo } \\
\text { berechnetes } \\
\text { Knallgas rolumen }\end{array}$ & $\begin{array}{c}\text { Eutwickeltes Gas } \\
+ \text { disponibler } \\
\text { Sauerstof in der } \\
\text { Ueberschwefel- } \\
\text { sanre }\end{array}$ & Defficit & $\begin{array}{l}\text { Znnahme } \\
\text { des Defieits } \\
\text {, }\end{array}$ \\
\hline $1 / 2 \mathrm{St}$ & 6,87 cem & 5,83 & 1,04 & 1,04 \\
\hline $1 "$ & $13,74 \quad$ & 11,87 & $1,8 i$ & 0,83 \\
\hline $11 / 2 n$ & 20,61 " & 18,29 & 2,32 & 0,45 \\
\hline
\end{tabular}

Wenn das Deficit von Reduction der Ueberschwefelsäure herrührte, müsste es bei wachsender Zeit in beschleunigtem Maasse zunehmen, da der Gehalt der Flüssigkeit wächst. Es nimmt aber in verzögertem Maasse $z u$, wie dies von der Absorption zu erwarten ist.

Aus diesen Versuchen und dem Umstande, dass für die kleineren Intensitäten das Deficit annähernd dasselbe ist, während der Gehalt der Flüssigkeit an Ueberschwefelsäure ausserordentlich verschieden ist, kann min wohl schliessen, dass das Deficit für die kleineren Intensitäten lediglich auf Absorption zurückzuftuhren ist. Unzweifelhaft rührt aber bei grösseren Intensitäten ein guter Theil des Deficits von Reduction der Ueberschwefelsäure her. Columne V der Tabelle anf p. 203 gibt den Bruchtheil der aus der Tangentenbussole berechneten Knallgasmenge an, welcher als disponibler Sauerstoff der Ueberschwefelsäure entwickelt ist, wenn das Deficit gar nicht in Rechnung gezogen wird; Columne VI denselben bei den grösseren Intensitäten, wenn das Deficit als ganz von reducirter Ueberschwefelsăure herrührend angenommen wird. Beide Zahlenreihen haben denselben Verlauf. Wie bemerkt, sind sie den Mengen Ueberschwefelsäure proportional, welche 
ceteris paribus gebildet würden, wenn die mittlere Stromdichtigkeit auf der positiven Electrode den Zahlen der Columne I auf p. 203 proportional wäre. In Tafel III Fig. 4 sind die Zahlen der Columne VI für grössere, die der Columne V für kleinere Intensitäten multiplicirt mit 1000 als Ordinaten zu den Zahlen der Columne I als Abscissen abgetragen.

Ueber den Einfluss der Stromdichtigkeit sagt G. W i ede $\operatorname{man} n^{\left.\frac{1}{)}\right)}$ :

,Vermehrung der Stromesdichtigkeit, also Vergrösserung der Intensität und Verkleinerung der Obertläche der positiven Platinelectrode, vermehrt die Menge jener Substanzen so lange, als nicht die dadurch bewirkte Temperaturerhöhung der Electrode sie wieder vernichtet. Dies liegt darin, dass bei einer grösseren Stromesdichte dieselben in der gleichen Zeit auch in grösserer Menge an der Platinelectrode erscheinen, und so durch die katalytische Wirkung des Platins weniger schnell zerstört werden. Zugleich würde indes hierbei auch der gebildete Sauerstoff in weniger innige Berührung mit dem $W$ asser und der Säure kommen und unverändert entweichen. Es muss also eine mittlere Stromesdichtigkeit geben, bei der die Sulistanzen sich in grösster Menge bilden."

Meine Versuche bestätigen diese Vermuthung in Bezug auf die Ueberschwefelsäure rollkommen. Bei den grőssten benutzten Intensitäten machte sich die Erwärmung auch bereits durch das schnelle Abschmelzen des Eises um das Zersetzungsgefäss bemerkbar; bis das Eis wieder angedrückt wurde, stieg die Intensität infolge der vermehrten Leitungsfähigkeit der Säure; Regulirung der Intensität und Constanthalten der Temperatur wird infolgedessen bei starken Strömen schwierig.

Es findet nicht gleichzeitig mit dem Maximum für die Ueberschwefelsäure auch ein solches fur das Ozon statt; sondern, wie die letzte Rubrik der auf p. 203 unten zusammen1883.

1) G. Wiedemann, Die Lehre von der Electricität, 2. p. 552. 
gestellten Zahlen zeigt, nehmen die Mengen des gebildeten Ozons mit wachsender mittlerer Stromdichtigkeit noch weiter zu. In der That hat auch beim Ozon die weniger innige Berührung des Sauerstoffes mit der Flüssigkeit bei grosser Stromdichtigkeit eher einen günstigen als schädlichen Einfluss. Vielleicht findet aber infolge der Temperaturerhöhung der Electrode bei sehr grosser Stromdichtigkeit auch noch ein Maximum für das Ozon statt.

VII. Einfluss der Concentration der saure.

Um den Einfluss der Concentration der Säure auf die Mengen der entwickelten Superoxyde zu untersuchen, wurde eine Reihe ron Versuchen stets bei derselben Intensität und von einer Viertelstunde Dauer des Stromschlusses mit Mischungen von verschiedenem Procentgehalt angestellt. Die aus der Ablenkung der Tangentenbussole berechnete Knallgasmenge war jedesmal $90,55 \mathrm{ccm}$. Der gesammte disponible Sauerstoff in der Flüssigkeit wurde durch Titriren eines bestimmten Bruchtheiles derselben mit Eisenvitriol, das Wasserstoffsuperoxyd durch Titriren eines gleichen Theiles mit ubermangansaurem Kali bestimmt.

Es ergaben sich folgende Zahlen:

\begin{tabular}{|c|c|c|c|c|}
\hline \multirow{2}{*}{$\begin{array}{l}100 \text { Gewiebtstheile } \\
\text { der Szare ent- } \\
\text { halten } \mathrm{H}_{3} \mathrm{SO}_{4}\end{array}$} & \multicolumn{3}{|c|}{ Disponibler Sauerstoff } & \multirow{2}{*}{$\begin{array}{c}\begin{array}{c}\text { Deficit an } \\
\text { elogtrolytisobeu } \\
\text { Producten }\end{array} \\
\end{array}$} \\
\hline & $\begin{array}{c}\text { dea entwickelten } \\
\text { Ozons }\end{array}$ & $\begin{array}{c}\text { des Geha!ts der } \\
\text { Ueberehwefel- } \\
\text { seure }\end{array}$ & 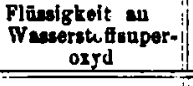 & \\
\hline $\begin{array}{l}10,1 \\
19,8 \\
28,3 \\
39,5 \\
50,7 \\
60,0 \\
69,4 \\
77,6 \\
89,4\end{array}$ & $\begin{array}{l}0,11 \\
0,18 \\
0,11 \\
0,10 \\
0,15 \\
0,06 \\
0,05 \\
0,07 \\
0,07\end{array}$ & $\begin{array}{r}0,62 \\
6,79 \\
16,25 \\
22,01 \\
18,76 \\
4,50 \\
3,49 \\
2,55 \\
1,21\end{array}$ & $\begin{array}{c}0 \\
0 \\
0 \\
0 \\
0 \\
2,54 \\
3,43 \\
4,17 \\
2,61\end{array}$ & $\begin{array}{l}1,65 \\
1,94 \\
2,28 \\
2,17 \\
1,84 \\
1,65 \\
0,86 \\
0,17 \\
0,31 .\end{array}$ \\
\hline
\end{tabular}

Das Deficit ist, wie immer, wieder zum Theil durch $\mathbf{R e}_{e}$ duction ron Ueberschwefelsäure, resp. Wasserstoffsuperoxyd, zum Theil durch Absorption von Knallgas hervorgerufen. Sein Maximum fällt ungefähr mit dem Maximum für die Ueberschwefelsäure zusammen, bei $30-40$ procentiger Säure. 
Vom Maximum ausgehend nimmt für geringere Concentrationen das Deficit langsamer, für höhere etwa im gleichen Verhältniss ab, wie der disponible Sauerstoff in der Flüssig. keit. Die Absorption des Knallgases scheint also bei stark concentrirter Säure geringer zu sein, als bei rerdünnter. Für den Verlauf unserer Zahlen ist es auch hier wieder gleichgültig, ob ich $1 / 3$ des Deficits als Correction wegen der Reduction zur gemessenen Zahl der Ueberschwefelsäure eventuell vertheilt auf die der Ueberschwefelsäure und des Wasserstoffsuperoxyds - hinzufüge, oder nicht.

Die Zahlen für das entwickelte Ozon leiden wie stets an grosser Unsicherheit. Nur das ist mit Sicherheit zu folgern, dass bei grösseren Concentrationen ron $60 \%$ aufwärts die Ozonmengen kleiner sind, als bei niedrigeren Concentrationen. Für die Ueberschwefelsäure findet ein Vaximum bei etwa 40 procentiger Säure statt. Von den $30,18 \mathrm{ccm}$ Sauerstoffgas, welche nach Angabe der Tangentenbussole entwickelt werden sollten, erscheinen bei 40 procentiger Säure $22,01 \mathrm{ccm}$, also fast drei Viertel in Form disponiblen Sauerstoffes der Ceberschwefelsäure. Es ist dies das grösste Verhältniss, welches bei meinen sämmtlichen Versuchen erreicht wurde.

C. Hoffmann hat bereits in der eingangs erwähnten Abhandlung die Ozonmengen und den disponiblen Sauerstoff in der Flüssigkeit für Mischungen ron $40 \mathrm{Vol}$. Wasser zu $1 \mathrm{Vol} . \mathrm{H}_{2} \mathrm{SO}_{4}$ bis auf $3 \mathrm{Vol}$. Wasser zu $1 \mathrm{Vol}$. $\mathrm{H}_{3} \mathrm{SO}_{4}$ bestimmt. Für das Ozon fand er ein Maximum bei der Säure mit 5 Vol. Wasser, die Menge des disponiblen Sauerstoffes in der Lösung fand er mit wachsender Concentration fortwährend zunehmend. Seine Zahlen sind in Milligrammen:

\begin{tabular}{|c|c|c|c|c|c|}
\hline \multirow{2}{*}{$\begin{array}{c}\text { Procentgehalt } \\
\text { der Saure (Ge- } \\
\text { wichtuproc.) }\end{array}$} & \multicolumn{2}{|c|}{ Disponibler Sauerstoff } & \multirow{2}{*}{$\begin{array}{l}\text { 'Procentgehalt } \\
\text { der Sinre (Go- } \\
\text { wichtepr 0c.) }\end{array}$} & \multicolumn{2}{|c|}{ Disponibler Sauerstoff } \\
\hline & im O2on & 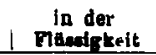 & & Im Ozon & $\begin{array}{c}\text { in der } \\
\text { Flïentrkeit }\end{array}$ \\
\hline $\begin{array}{r}4,4 \\
8,5 \\
10,9 \\
15,6 \\
18,7\end{array}$ & $\begin{array}{l}0,04 \\
0,28 \\
0,32 \\
0,36 \\
0,36\end{array}$ & $\begin{array}{l}0,12 \\
0,24 \\
0,84 \\
1,25 \\
2,0 \pm\end{array}$ & $\begin{array}{l}28,3 \\
26,9 \\
31,6 \\
38,0\end{array}$ & $\begin{array}{l}0,40 \\
0, \pm 4 \\
0,40 \\
0,23\end{array}$ & $\begin{array}{l}2,52 \\
2,68 \\
4,28 \\
6,00\end{array}$ \\
\hline
\end{tabular}


Mit einem passenden Factor multiplicirt sind die Hoffmann'schen Zahlen für die Ueberschwefelsäure neben den meinigen in Tafel III Fig. 5 abgetragen. Sie zeigen einen weit unregelmässigeren Verlauf, als diese. Es kann das nicht wundern, wenn man bedenkt, dass Hoffmann bei seinen Versuchen alle übrigen Bedingungen, ausser Temperatur und Dauer des Stromschlusses nur insofern constant erhielt, als er jedesmal dieselbe Anzahl Elemente nahm. Es blieben also veränderlich: Vertheilung der Stromdichtigkeit auf der positiven Electrode, die Intensität bei verschiedenen Concentrationen infolge der verschiedenen Leitungsfähigkeit der Säure und die Intensität während der Dauer eines Versuches.

Wasserstotfsuperoxyd tritt, wie meine Versuche zeigen, erst ron etwa $60 \%$ an in der Flüssigkeit auf. Die Menge desselben erreicht bei 80 procentiger Säure ihr Maximum. Das Verhältniss von Wasserstoffsuperoxyd zu Ueberschwefelsäure nimmt mit wachsender Concentration zu. Soweit dies bei der Verschiedenheit der Methoden zu erwarten ist, stimmen meine Versuche für höhere Concentrationen mit denen Berthelot's uberein. Berthelot ${ }^{1}$ ) hatte die positive Electrode mit einem porösen Thongefäss umgeben. Er liess den Strom einer Anzahl Elemente mehrere Tage hindurch geschlossen. Infolge der electrischen Osmose und der Wanderung der Schwefelsäure wurde das Flüssigkeitsvolumen im Thoncylinder immer kleiner und concentrirter, die Stromintensität immer geringer. Von Zeit zu Zeit titrirte er ein kleines, mit der Pipette herausgenommenes Flüssigkeitsvolumen, welches er durch frische Säure ersetzte. Das erste Auftreten von Wasserstoffsuperoxyd constatirte er, wenn die Säure etwa die Zusammensetzung $\mathrm{H}_{2} \mathrm{SO}_{4}+4 \mathrm{H}_{2} \mathrm{O}$ hatte ${ }^{2}$ ), was 57,7 Gewichtsprocenten entspricht. Er fand ferner, dass sich das Verhältniss ron Wasserstoffsuperoxyd und Ueberschwefelsäure bei längerer Dauer des Stromschlusses dem Werthe 2 näherte, welcher nie uberschritten wurde, und

1) Berthelot, Compt. rend. 90. p. 269. 1880.

2) Berthelot, l. c. p. 270. 
welcher sich auch bei der freiwilligen Zersetzung der sich selbst überlassenen Flüssigkeit erhielt. Er nimmt deshalb die Existenz einer Verbindung $\mathrm{S}_{2} \mathrm{O}_{7}+2 \mathrm{H}_{2} \mathrm{O}_{2}$ an. Es scheint auch aus meinen Versuchen herrorzugehen, dass sich bei wachsender Concentration jenes Verhaltniss einstellt. Meine Versuche über $90 \%$ hinaus fortzusetzen, wäre sehr schwierig gewesen, da infolge der geringen Leitungsfähigkeit der concentrirten Säure bei $90 \%$ bereits 17 Grove'sche Becher erforderlich waren, um die gewünschte Intensität zu erhalten, und $\mathrm{da}$ das die Zersetzungszelle umgebende Eis infolge der Erwärmung der Säure durch den Strom sehr schnell ab. schmolz. Auch würde bei höheren Concentrationen die Bildung von schwefliger Säure an der Wasserstoffelectrode die Versuche gestört haben.

In Bezug auf die Bildung der Superoxyde bei der Electrolyse verdünnter Schwefelsäure zwischen Platinelectroden bleibt noch die wichtige Frage unerledigt, wie sich die electromotorische Kraft der Polarisation durch dieselbe ändert. Ich hoffe, dass es mir auf Grund der vorliegenden Arbeit gelingen wird, die Antwort auf diese Frage zu geben.

\section{Antwort auf einige Bemerkungen des Herrn F. Kohlrausch; von $\mathrm{H}$. Wild.}

In der ausführlichen Darlegung meiner „Bestimmung des Werthes der Si emens'schen Widerstandseinheit in absolutem, electromagnetischem Maasse" habe ich einleitend die Punkte hervorgehoben, in welchen meine Ausfuhrung dieser Bestimmung nach der zweiten Weber'schen Methode (3. Methode gemäss der Bezeichnung von G. Wiedemann) sich von denjenigen nach derselben Methode vor mir von anderen gemachten Bestimmungen unterschied. Es schien mir dies geboten, um dem Leser für den Fall, dass mein Resultat Ann. d. Phy, a. Chem. N. F. IXIV. 


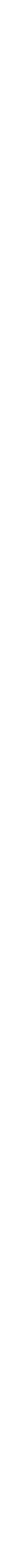

